\title{
A case of Vaccine-Induced Thrombotic Thrombocytopenia (VITT) with thrombosis of the superior mesenteric artery after ChAdOx1 nCov-19 COVID-19 Vaccine
}

\author{
Laura Pini, ${ }^{1,2 *}$ Paolo Malerba, ${ }^{2,3 *}$ Jordan Giordani, ${ }^{1}$ Deborah Stassaldi, ${ }^{2}$ Carlo Aggiusti, ${ }^{2}$ \\ Giuliana Martini, ${ }^{4}$ Giulia Arenare, ${ }^{2,3}$ Chantale Premoli, ${ }^{2,5}$ Giulia Bosio, ${ }^{2,5}$ Marco Lancelotti, ${ }^{2,5}$ \\ Francesca Bosisio, ${ }^{2,5}$ Giorgia Muzi, ${ }^{2,5}$ Maria Lorenza Muiesan ${ }^{2}$ \\ ${ }^{1}$ Respiratory Medicine Unit; ${ }^{2}$ UOC 2 a Medicina, Spedali Civili di Brescia, Department of Clinical and \\ Experimental Sciences, University of Brescia, Brescia; ${ }^{3}$ Post-graduate School of Internal Medicine, \\ University of Brescia, Brescia; ${ }^{4}$ Hemostasis Central Laboratory, ASST Spedali Civili of Brescia, Brescia; \\ ${ }^{5}$ Post-graduate School of Emergency Medicine, University of Brescia, Brescia, Italy
}

\begin{abstract}
We describe the case of a 64 years-old woman admitted to the Emergency Department of the ASST-Spedali Civili of Brescia with fever and gastrointestinal symptoms 10 days after the first dose of ChAdOx1 nCov-19 vaccine. Laboratory investigations showed severe thrombocytopenia and high D-dimer, while an abdominal CT
\end{abstract}

Correspondence: Laura Pini, Respiratory Medicine Unit, Spedali Civili di Brescia, Department of Clinical and Experimental Sciences, University of Brescia, Piazzale Spedali Civili 1, Brescia, Italy.

Tel.: +39.(0)30.399.6263

E-mail: laura.pini@unibs.it

Key words: Vaccine induced thrombotic thrombocytopenia; SARSCoV2; COVID-19 Vaccine; ChAdOx1 nCov-19; AstraZeneca.

Contributions: LP and PM equally contributed to this paper. CA, LP, DS, GM, PM, GA, CP, GB, ML, FB, GM: clinical case management; PM, JG, PM, GA, CP, GB, ML, FB, GM: clinical data collection; PM, JG, GA: first draft; MLM: manuscript revision and chief of the department.

Conflict of interests: The authors declare no conflict of interest.

Availability of data and materials: All data underlying the findings are fully available.

Ethics approval and consent to participate: No ethical committee approval was required for this case report by the Department, because this article does not contain any studies with human participants or animals. Informed consent was obtained from the patient included in this study.

Consent for publication: The patient gave her written consent to use her personal data for the publication of this case report and any accompanying images.

Received for publication: 12 August 2021.

Revision received: 28 October 2021.

Accepted for publication: 9 November 2021.

This work is licensed under a Creative Commons Attribution 4.0 License (by-nc 4.0).

COPyright: the Author(s), 2021

Licensee PAGEPress, Italy

Emergency Care Journal 2021; 17:10032

doi:10.4081/ecj.2021.10032 scan reported a partially occluding thrombosis of the superior mesenteric artery. Following treatment with fondaparinux, immunoglobulins and high dosage steroid therapy, we observed a rapid improvement of patient's conditions. An ELISA assay showed the presence of antibodies against heparin PF4-complex. Subsequent abdominal CT-scan showed the superior mesenteric artery thrombosis resolution, and the patient was finally discharged after 12 days of hospitalization. Several reports pointed to the venous system as the main district affected by Vaccine-Induced Thrombotic Thrombocytopenia (VITT), while a peculiar feature of this report is the involvement of the mesenteric arterial system. Further investigation of VITT's pathophysiological mechanisms is mandatory to develop preventive strategies and effective treatments.

\section{Introduction}

To counteract the devastating consequences of the SARS-CoV2 pandemic, major efforts and resources have been employed worldwide to research and develop therapies to address the acute and sub-acute phases of the disease and prevent further infection spreads.

Different therapeutical protocols and treatment strategies are available to limit the broad spectrum of clinical consequences of the infection, ranging from interstitial pneumonia to thromboembolic events. ${ }^{1-4}$

Preventive measures represent another trivial point in the containment of SARS-CoV2 spreading. It is undeniable that the development of effective vaccines within a very short timeframe represented a landmark achievement. ${ }^{5}$ Several vaccination plans are currently available worldwide. According to the portal Our World in Data developed by the Oxford Martin School/University of Oxford, as of October $26^{\text {th }} 2021,48,7 \%$ of the world's population has received at least one dose of vaccine. Data from the Italian Government - Health Ministry report that in Italy, $86 \%$ of the population over- 12 has received at least one dose of vaccine, and $82 \%$ completed the vaccination program.

The efficiency and progress of the vaccination campaign rely on the different vaccine's availability and indications, as established by the European Medicines Agency (EMA). There are currently four vaccines authorized and regulated for use in Europe. The first two, "BNT162B2" by Pfizer/BioNTech and "mRNA-1273" by Moderna, rely on an innovative mRNA technology encoding for the viral spike (S) protein, encapsulated into lipid nanoparticles. Other two 
vaccines, AstraZeneca's ChAdOx1 nCov-19 and Johnson \& Johnson/Janssen's Ad26.COV.2 are based on an adenoviral vector codifying for the spike protein antigen. ${ }^{6}$

Like any drug, SARS-CoV2 vaccines have different side effects, with pain at the injection site, polyarthralgia, fever with chills, fatigue and gastrointestinal symptoms occurring most frequently. ${ }^{7}$

In this context, there have been heated discussions and controversial arguments due to the occurrence of thromboembolic events following the administration of ChAdOx1 nCov-19 and Ad26.COV.2 vaccines. ${ }^{8,9}$

This paper reports a clinical case of a Vaccine-Induced Thrombocytopenic Thrombosis (VITT) involving the superior mesenteric artery in a 64-year-old woman who received the first dose of ChAdOx1 nCov-19 vaccine 10 days before admission.

\section{Case Report}

A 64 years-old woman was admitted on May $10^{\text {th }}, 2021$ to the Emergency Department (ED) of the ASST-Spedali Civili in Brescia (Northern Italy), complaining of fever $\left(\mathrm{T} \max 38^{\circ} \mathrm{C}\right)$, fatigue, abdominal pain, vomiting unresponsive to metoclopramide, and diarrhea (5-6 episodes/day). She had received the first dose of ChAdOx1 nCov-19, AstraZeneca vaccine against SARS-CoV2, on April $30^{\text {th }}$. Her past medical history included hypercholesterolemia in treatment with statins and gastro-esophageal reflux disease, and she did not report personal or family history of venous thromboembolism.

Clinical parameters at ED arrival were: heart rate (HR) $55 \mathrm{bpm}$, blood pressure (BP) 180/85 $\mathrm{mmHg}$, peripheral arterial oxygen saturation $\left(\mathrm{SpO}_{2}\right) 99 \%$ and tympanic temperature $35.5^{\circ} \mathrm{C}$.

Clinical evaluation showed a Glasgow Coma Scale (GCS) value of 15 without other significant clinical or neurological findings. Laboratory investigations showed leukocytosis $\left(12.57 \times 10^{3} / \mu \mathrm{L}\right)$ with neutrophilia $\left(10.44 \times 10^{3} / \mu \mathrm{L}\right)$, thrombocytopenia $(45.000 / \mu \mathrm{L})$, mild hypokalemia $(3.0 \mathrm{mmol} / \mathrm{L})$ and a mild increase in hepatic (LDH: 315U/L - AST: 49U/L) and systemic inflammatory indexes (CRP: 17.6mg/L - Ferritin: $198 \mathrm{ug} / \mathrm{L}$ ). PT was $13.3 \mathrm{sec}$, PT ratio 1.1, APTT $24.3 \mathrm{sec}$, APTT ratio 0.79 with Fibrinogen $81 \mathrm{mg} / \mathrm{dL}$ and a marked increase in D-dimer ( $>35000 \mathrm{ng} / \mathrm{mL})$. A nasopharyngeal swab for SARS-CoV2 infection tested negative.

While in the ED, the patient complained of a minor episode of hematemesis with rapid resolution. A whole brain, chest, and abdomen Computerized Tomography (CT)-scan with and without contrast medium (lopamidol 370, 110mL) was then performed. CT scan of the brain and chest did not highlight any significant finding and excluded the presence of pulmonary embolism, while abdominal scans indicated a $2 \mathrm{~cm}$ long partially occluding thrombosis of the superior mesenteric artery, with local thickening and hyperemia of small intestine loops (Figure 1). Abdominal scans further identified two small non occluding thrombi at the origin of portal branches to the V and VII hepatic segments and two small areas of hypoperfusion at the cortical level of both kidneys, suggestive of ischemic lesions. A hematological evaluation hypothesized an immune-mediated nature of the thrombosis and thrombocytopenia observed, and the diagnosis of Vaccine-Induced Thrombotic Thrombocytopenia (VITT) was assumed due to the recent administration of ChAdOx $1 \mathrm{nCov}-19$ vaccine. Therefore, the patient was admitted to the Internal Medicine Department (U.O.C. Medicine 2) for a further diagnostic and therapeutic workup.

Treatment included high dosage steroids with methylprednisolone $(1 \mathrm{mg} / \mathrm{kg} / \mathrm{die})$ for one day followed by dexamethasone $(0.6 \mathrm{mg} / \mathrm{kg} / \mathrm{die})$ for the subsequent two days and immunoglobulins $(500 \mathrm{mg} / \mathrm{kg} / \mathrm{die}-30 \mathrm{~g} / \mathrm{die}$ in total) for a total of three days. Subcutaneous anticoagulant therapy with fondaparinux was also started at $7.5 \mathrm{mg} /$ die dosage, considering the presence of arterial thrombosis. Thrombocytopenia screening included viral hepatitis antigens, HIV, anti-cardiolipin, and anti-B2GP1 antibodies, antithrombin, Lupus Anti-Coagulant (LAC), Protein C and S, Antinuclear Antibodies (ANA), Extractable Nuclear Antigen Antibodies (ENA) and all tested negative. Screening for mutation G20210A and V617F of prothrombin and JAK2 genes, respectively, were also negative, while an ELISA assay highlighted the presence of antibodies against heparin PF4-complex, subsequently verified with a positive HIPA test, thus confirming the diagnosis of VITT.

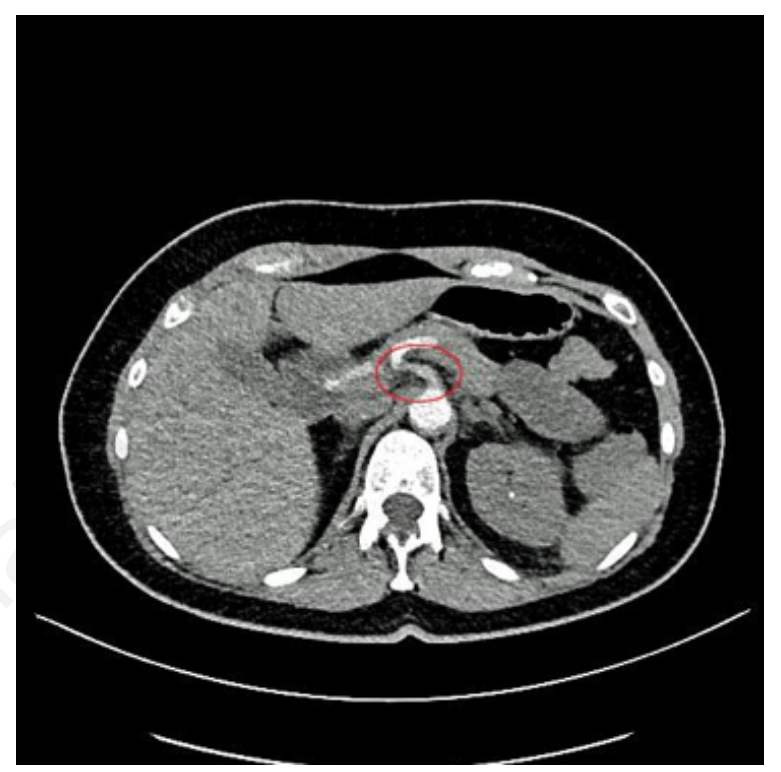

Figure 1. Abdominal CT-Scan frame showing a non-occluding thrombosis of the Superior Mesenteric Artery.

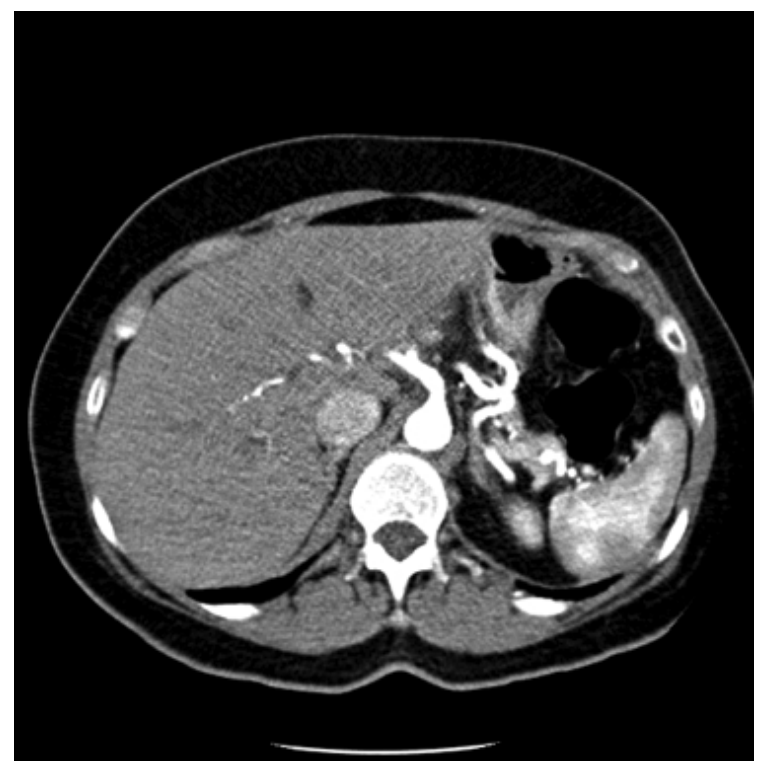

Figure 2. Abdominal CT-Scan frame, repeated on day 4, showing resolution of the Superior Mesenteric Artery thrombosis previously described. 
On day 2, the patient complained of severe occipital headache, and a head CT scan was repeated, showing no acute lesions. She also presented a self-limiting, minor episode of melena on day 3 with a slight decrease in hemoglobin level. At esophagogastroduodenoscopy, erosive antral gastritis without signs of acute bleeding was described. On day 4, a CT abdominal angiography was performed, showing an almost complete resolution of the mesenteric thrombosis previously described (Figure 2) and the persistence of portal branches' small thrombotic defects. We observed a progressive increase in platelet count, reaching a complete restoration on day $6(167.000 / \mu \mathrm{L})$. Due to the persistence of hypofibrinogemia, we hypothesized a congenital fibrinogen defect; patient's first-grade relatives, therefore, underwent quantitative fibrinogen laboratory dosage showing normal results. Patient's fibrinogen levels increased in the subsequent days, reaching normal values on day 8 .

Following a progressive, gradual improvement of clinical conditions, the patient was discharged on day 13. She was in good clinical condition, afebrile, eupnoic and hemodynamically stable. Laboratory testing at discharge showed: platelets $224000 / \mu \mathrm{L}, \mathrm{Hb}$ $12.2 \mathrm{mg} / \mathrm{dL}$, fibrinogen $510 \mathrm{mg} / \mathrm{dL}$ and CRP $2 \mathrm{mg} / \mathrm{L}$. Treatment with warfarin for 3 months was prescribed at discharge. At follow-up visit after one month, the patient described a further improvement in general health conditions. Good adherence to therapy with warfarin was confirmed by INR dosage.

\section{Discussion}

This case report describes a rare case of vaccine-induced thrombotic thrombocytopenia with both arterial and venous thrombosis following administration of AstraZeneca's ChAdOx1 nCov-19 vaccine. According to the German Society of Thrombosis and Haemostasis (GTH) studies, VITT accounts for 13 cases out of 1.6 million doses of administered vaccine. ${ }^{10}$

Our case is of particular interest for simultaneous arterial and venous vascular districts' involvement and patient's age. Several reports of thrombotic events following the administration of ChAdOx1 nCov-19 vaccine have been described, but in most of them, venous vessels are mainly involved, particularly the intracranial venous sinuses and the splanchnic venous vessels. ${ }^{11,12}$ Our patient's age also represents an unusual finding compared to previous cases reported in literature, as higher risk subjects for vaccine-associated thrombotic events are usually women younger than 50 years of age and without significant past medical history. ${ }^{10}$

Due to initial gastrointestinal ischemia, the patient's symptoms at presentation are different from those usually associated with venous thrombosis, further challenging the diagnosis. In this context, recommendations provided by different scientific societies as the International Society on Thrombosis and Haemostasis (ISTH) and the Italian Society for the Study of Haemostasis and Thrombosis (SISET), as well as case reports and data from the literature indicated the need for laboratory testing characteristics to confirm the presence of VITT, i.e. thrombocytopenia, hypofibrinogemia and increased D-Dimer, all suggestive of a consumption coagulopathy. ${ }^{13-}$ 16

\section{Conclusions}

Besides describing an interesting clinical case of an uncommon variant of a known infrequent SARS-CoV2 vaccine-related side effect, this case report would like to raise two further messages.

Knowledge of vaccine's side effects, clinical manifestations, and management may help health workers to reassure the population about risks and adverse reactions, thus ensuring that the mass campaign vaccination continues with even greater adherence. In this regard, it is necessary to ensure continuous, up-to-date and appropriate information to the different population cohorts to increase the awareness of the importance and usefulness of vaccination as the only effective current way of preventing the spread of SARS-CoV2 infection. ${ }^{8,17}$

On the other hand, studies aimed to characterize the VITT's clinical and molecular mechanisms are warranted. Several interesting pathogenic and therapeutic similarities have already been identified between VITT and the Heparin-Induced Thrombocytopenia (HIT) due to the presence in both diseases of autoantibodies targeting the heparin-Platelet Factor 4 (PF4) complex. The derived immune complexes lead to intense intravascular platelet aggregation, thrombus formation and rapid subsequent platelet count reduction. ${ }^{6,18-20}$

The rapid identification of such an important analogy between two diseases with different origins should encourage further research in this field with dedicated attention to the rarest effects of vaccination therapy.

\section{References}

1. Sabeerabi B, Vemula S, Vadde R, Nagaraju GP. COVID-19: Where is the treatment? World J Clin Oncol 2021;12:309-22.

2. Orzes N, Pini L, Levi G, et al. A prospective evaluation of lung function at three and six months in patients with previous SARS-COV-2 pneumonia. Resp Med 2021;106541

3. Quek E, Tahir H, Kumar P, et al. Treatment of COVID-19: a review of current and prospective pharmacotherapies. Br J Hosp Med (Lond) 2021;82:1-9.

4. Heustess AM, Allard MA, Thompson DK, Fasinu PS. Clinical management of COVID-19: A review of pharmacological treatment options. Pharmaceuticals (Basel) 2021;14:520.

5. Sharma O, Sultan AA, Ding H, Triggle CR. A review of the progress and challenges of developing a vaccine for COVID19. Front Immunol 2020;11:585354.

6. Rzymski P, Perek B, Flisiak R. Thrombotic thrombocytopenia after COVID-19 vaccination: In search of the underlying mechanism. Vaccines (Basel) 2021;9:559.

7. Doroftei B, Ciobica A, Ilie OD, et al. Mini-Review Discussing the Reliability and Efficiency of COVID-19 Vaccines. Diagnostics (Basel) 2021;11:579.

8. Long B, Bridwell R, Gottlieb M. Thrombosis with thrombocytopenia syndrome associated with COVID-19 vaccines. Am J Emerg Med 2021;49:58-61.

9. Lavin M, Elder PT, O'Keeffe D, et al. Vaccine-induced immune thrombotic thrombocytopenia (VITT) - a novel clinicopathological entity with heterogeneous clinical presentations. Br J Haematol 2021;195:76-84.

10. Oldenburg J, Klamroth R, Langer F, et al. Diagnosis and management of vaccine-related thrombosis following AstraZeneca COVID-19 vaccination: Guidance statement from the GTH. Hamostaseologie 2021;41:184-9.

11. Gras-Champel V, Liabeuf S, Baud M, et al. Atypical thrombosis associated with VaxZevria ${ }^{\circledR}$ (AstraZeneca) vaccine: Data from the French Network of Regional Pharmacovigilance Centres. Therapie 2021:S0040-5957(21)00130-X. 
12. Pottegård A, Lund LC, Karlstad Ø, et al. Arterial events, venous thromboembolism, thrombocytopenia, and bleeding after vaccination with Oxford-AstraZeneca ChAdOx1-S in Denmark and Norway: population based cohort study. BMJ 2021;373:n1114.

13. ISTH Interim Guidance for the Diagnosis and Treatment on VaccineInduced Immune Thrombotic Thrombocytopenia. Available from: https://www.isth.org/page/covid19

14. Gresele P, Marietta M, Ageno W, et al. Management of cerebral and splanchnic vein thrombosis associated with thrombocytopenia in subjects previously vaccinated with Vaxzevria (AstraZeneca): a position statement from the Italian Society for the Study of Haemostasis and Thrombosis (SISET). Blood Transfus 2021;19:281-3.

15. Franchini M, Liumbruno GM, Pezzo M. COVID-19 vaccineassociated immune thrombosis and thrombocytopenia (VITT): Diagnostic and therapeutic recommendations for a new syndrome. Eur J Haematol 2021;107:173-80.

16. Karnam A, Lacroix-Desmazes S, Kaveri SV, Bayry J. Vaccineinduced prothrombotic immune thrombocytopenia (VIPIT): Consider IVIG batch in the treatment. J Thromb Haemost 2021;19:1838-9.

17. Hunter PR. Thrombosis after covid-19 vaccination. BMJ 2021;373:n958.

18. Scully M, Singh D, Lown R, et al. Pathologic antibodies to platelet factor 4 after ChAdOx1 nCoV-19 vaccination. N Engl J Med 2021;384:2202-11.

19. Douxfils J, Favresse J, Dogné JM, et al. Hypotheses behind the very rare cases of thrombosis with thrombocytopenia syndrome after SARS-CoV-2 vaccination. Thromb Res 2021;203:163-71.

20. Patriquin CJ, Laroche V, Selby R, et al. Therapeutic Plasma Exchange in Vaccine-Induced Immune Thrombotic Thrombocytopenia. N Engl J Med 26;385:857-9. 